\title{
Magnetism and Superconductivity in Iron Pnictides
}

\author{
D.J. Singh \\ Materials Science and Technology Division, Oak Ridge National Laboratory \\ Oak Ridge, Tennessee 37831-6056, USA
}

\begin{abstract}
The discovery of high temperature superconductivity in iron pnictides and chalcogenides has resulted in surprising new insights into high temperature superconductivity and its relationship with magnetism. Here we provide an overview of some of what is known about these materials and in particular about the interplay of magnetism and superconductivity in them. Similarities and contrasts with cuprate superconductors are emphasized and the superconducting pairing is discussed within the framework of spin fluctuation induced pairing.
\end{abstract}

PACS: 74.70.Xa, 74.20.Rp, 74.20.Pq

\section{Background and introduction}

The discovery of superconductivity in a family of iron based compounds that are in proximity to magnetism $[1,2]$ has led to renewed interest in the interplay of these two phenomena in metals and many interesting discoveries.

Actually, the interplay of magnetism and superconductivity has a long history, starting with the Meissner effect and subsequently the Abrikosov flux lattice and type-II superconductivity. The development of the BCS theory of electron-phonon superconductivity led to understanding of the trends in the critical temperatures of the transition elements in terms of their electronic densities of states, $N\left(E_{\mathrm{F}}\right)$, and their Debye temperatures. Essentially, the Debye temperature sets the energy scale, and the electron-phonon interaction was proportional to $N\left(E_{\mathrm{F}}\right)$. This understanding led to the recognition of certain elements that did not fit the trends, most notably, $\mathrm{Pd}$, which is not superconducting, but does have a high density of states. This non-superconducting behavior was explained by Berk and Schrieffer in terms of the nearness of $\mathrm{Pd}$ to ferromagnetism [3].

The nearness of $\mathrm{Pd}$ to ferromagnetism comes also from its high density of states, as understood within the Stoner theory. Thus, the Fermi surfaces of the transition elements show two competing instabilities, one towards electron-phonon superconductivity, and the other towards ferromagnetism, both of which become enhanced as $N\left(E_{\mathrm{F}}\right)$ increases. Thus, ferromagnetism and superconductivity are competing instabilities. Clearly, the occurrence of these two phases "nearby" is a necessary condition for supposing that the superconductivity could be related to magnetism. However, as is clear from this historical example, such a proximity is by no means a sufficient condition, and in fact the superconductivity of transition metal elements is caused by the electron-phonon interaction. As discussed below, this is opposite to the case for the iron pnictides.
These Fe-based materials show both antiferromagnetism and superconductivity in their phase diagrams, with competition $[4,5]$. This at first sight may seem rather like the cuprates, but the magnetic phases are actually very different between the two classes of materials, as will be discussed below. One similarly is that in neither material can the superconductivity be explained within standard electron-phonon theory. This became apparent very quickly both from the fact that the phonon spectrum of LaFeAsO did not show the high phonon frequencies that would be needed to explain a high $T_{\mathrm{c}}[6]$, and from direct calculations that showed in addition that the electron-phonon coupling is weak [7].

\section{Structure and electronic structure}

The structural motif of the iron-pnictide and chalcogenide superconductors is the occurrence of Fe square planes with $\mathrm{Fe}$ in a nominally divalent state, and having a tetrahedral coordination by $\mathrm{P}, \mathrm{As}$, Se, Te or alloys including alloys with S (see Ref. [8] for a short review). This is illustrated along with the unit cell in Fig. 1. Aside from this common feature, a very wide variety of Fe-based superconductors have been discovered since the initial report of high $T_{\mathrm{c}}$ superconductivity in 2008. Here we focus on the features that are common to this family.

Density functional calculations $[6,9,10]$ confirmed that $\mathrm{Fe}$ is divalent in these compounds and showed an electronic structure with a rather high density of states (DOS) at the Fermi level, derived mainly from $\mathrm{Fe} d$ states, $N\left(E_{\mathrm{F}}\right) \approx 2 \mathrm{eV}^{-1}$ per Fe both spins depending on the material (see Fig. 2). This in itself places the compounds near itinerant magnetism. There is hybridization between the Fe $d$ states and the $p$ states of the coordinating ligands ( $\mathrm{As}, \mathrm{P}, \mathrm{S}, \mathrm{Se}, \mathrm{Te})$, but invariably the main ligand bands are located below the Fe $d$ bands generally at binding energies higher than $2 \mathrm{eV}$. This is in accord with spectroscopic experiments [11]. 
(a)

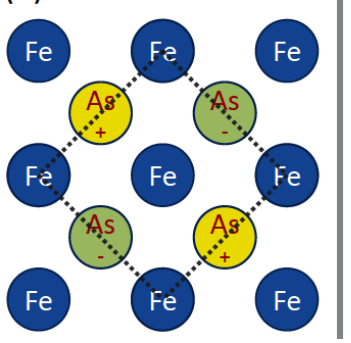

(b)

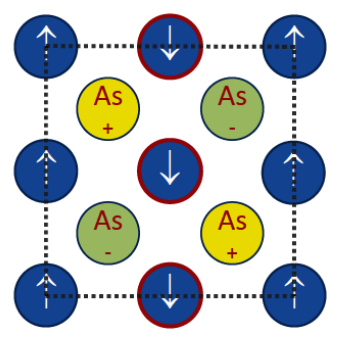

Fig. 1. (a) Structure of the FeAs layers in iron-pnictide superconductors. The Fe atoms are in a plane with As (or chalcogens) above (denoted + ) or below (denoted - ) to form a tetrahedral coordination. The dotted lines show the unit cell. Note that the cell contains two iron atoms. (b) Spin density wave magnetic structure. Note the doubling of the cell and the symmetry lowering from tetragonal to orthorhombic.

(a)

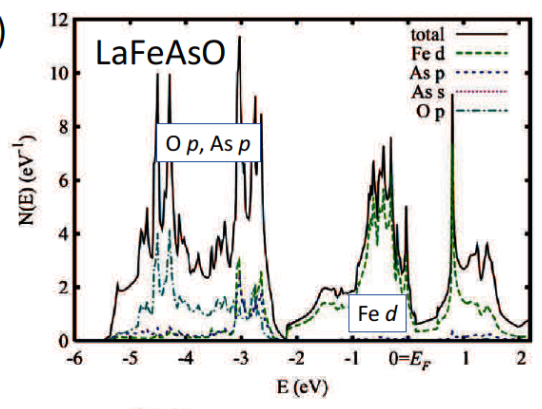

(b)

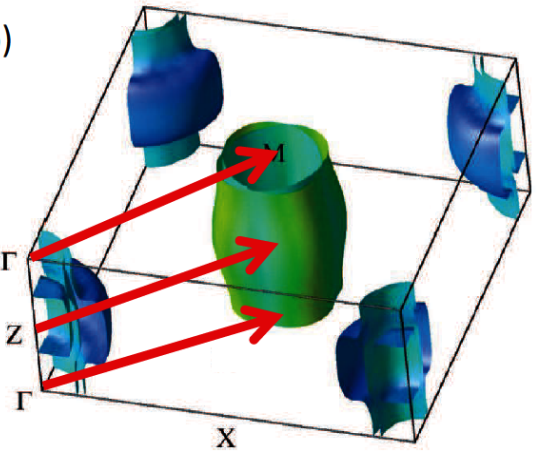

Fig. 2. (a) Density of states and projections of LaFeAsO as obtained in density functional calculations following Ref. [6]. (b) Fermi surface of LaFeAsO following Ref. [6]. The heavy arrows indicate the nesting of the electron and hole sheets. $\Gamma$ is the zone center, $(0,0,0), X$ is $(1 / 2,0,0), M$ is $(1 / 2,1 / 2,0)$ and $Z$ is $(0,0,1 / 2)$.

Turning to the shape of the Fe $d$ derived DOS, $E_{\mathrm{F}}$ in these compounds invariably lies on the lower energy side of a pronounced dip in the DOS. This dip is at an electron count of six $d$ electrons per Fe. In contrast, a tetrahedral crystal field scheme would split the Fe $d$ states into a lower lying $e_{\mathrm{g}}$ manifold containing four electrons and a higher $t_{2 \mathrm{~g}}$ manifold with six electrons. This difference between the actual shape of the DOS and the crystal field scheme reflects the importance of direct Fe-Fe hopping in forming the band structure, and is a consequence of the crystal structure, specifically the fact that the structure is built from edge sharing coordination polyhedra rather than the corner sharing octahedra characteristic of the cuprates. The Fe-Fe distance in the Fe-based superconductors is $\approx 2.8-2.9 \AA$, in contrast to the nearly $4 \AA$ in cuprates.

The DOS shows another key difference from cuprates. In cuprates, a single $d_{x^{2}-y^{2}}$ orbital plays the dominant role in the electronic structure near $E_{\mathrm{F}}$, while in the Fe-based superconductors, the $d$-shell is open and all the $d$ orbitals are involved. This is important from the point of view of the Mott physics. The multi-orbital nature of the low energy electronic structure opens channels for inter-orbital charge fluctuations that work against the Mott state. This was quantified by Gunnarsson and co-workers [12], who derived an approximate factor $1 / \sqrt{N}$, to be multiplied by $U / W$ when assessing the proximity of a material to a Mott transition (here $U$ is the effective Coulomb repulsion, i.e. the Hubbard $U, W$ is the band width and $N$ is the number of orbitals).

Within a strongly correlated picture, the effect of the Hubbard $U$ is generally to shift $d$ spectral weight away from the $E_{\mathrm{F}}$ to the Hubbard bands. This is the case in cuprates, but not in Fe-based superconductors, as was shown early on by X-ray absorption [13] and photoemission experiments [14]. There is a large $\mathrm{Fe} d$ spectral weight near the Fermi level, a renormalization of the $d$ bands (by a factor of $\approx 2$ ) and no Hubbard bands. This renormalization, which has been studied in detail by optical measurements, implies the presence of electronelectron correlation, but not the Mott physics normally associated with a large Hubbard $U$ [15]. What is seen is a rearrangement of the spectral weight within the $d$ bands, which can come both from the Hund rule coupling $J$ and the Hubbard parameter $U$ (see e.g. Ref. [16]), but no Hubbard bands. Importantly, in spite of the chemical diversity of this family of materials, no Mott insulating state has been found, strongly implying that the Fe-based superconductors are not in proximity to a Mott state. Instead the electronic structures appear to be more characteristic of intermetallic compounds than correlated oxides. One reflection of this is the fact that while alloying on the $\mathrm{Cu}$ site in cuprates is highly destructive to superconductivity, in the Fe-based materials high temperature superconductivity can be induced by alloying on the Fe site by other metals, such as Co in $\mathrm{BaFe}_{2-x} \mathrm{Co}_{x} \mathrm{As}_{2}$ and $\mathrm{SrFe}_{2-x} \mathrm{Co}_{x} \mathrm{As}_{2}[17,18]$, and even with replacement of as much as $40 \%$ of the Fe by $\mathrm{Ru}$ in $\mathrm{SrFe}_{2-x} \mathrm{Ru}_{x} \mathrm{As}_{2}$, $x=0.8[19]$.

\section{Fermi surface and nesting}

Superconductivity is fundamentally an instability of the Fermi surface. Interestingly, while the band structure is metallic and the density of states is high, the electronic structure at $E_{\mathrm{F}}$ consists of relatively small disconnected 
Fermi surfaces, as illustrated in Fig. 2. These are hole cylinders around the zone center, and electron cylinders around the zone corner.

The details vary between the different materials and as a function of doping. However, invariably there are two electron-like sections at the zone corner. These have the shape of two intersecting elliptical sections (see Fig. 2) that are derived from $d_{x z}, d_{y z}$ states on the inner part and $d_{x y}$ orbitals on the outer lobes. Also invariably, there are hole cylinders derived from $d_{x z}, d_{y z}$ orbitals at the zone center. There is also generally $k_{z}$-dependent participation of other orbitals, specifically the $d_{z^{2}}$ and $d_{x y}$ orbitals, at the zone center, either through hybridization or extra Fermi surface sheets. The hole sheets are generally heavier (lower Fermi velocity, less contribution to the conductivity) and more three-dimensional than the electron sheets. These basic features have been confirmed experimentally using angle-resolved photoemission (ARPES) by several groups working on the various materials [14, 20-25].

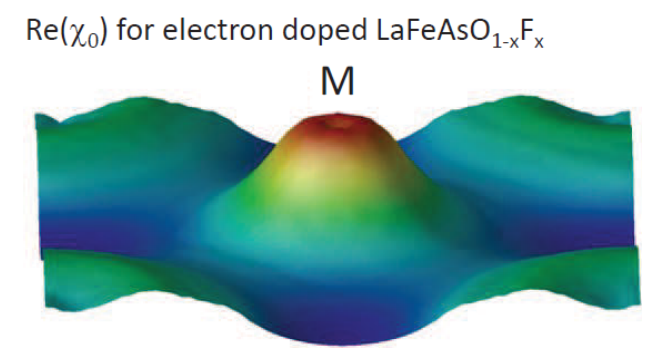

Fig. 3. Real part of the bare (non-interacting) susceptibility of electron doped LaFeAsO, following Ref. [26].

Since both the main electron and hole sheets share similar orbital character, and have similar shapes (cylinders), one can anticipate nesting. This in fact is the case as was shown by calculations of the Lindhard function [26]. Figure 3 shows the non-enhanced real part of the Lindhard function for electron doped LaFeAsO, following Ref. [26]. As may be seen, there is a broad peak of width comparable to the Fermi surface sizes, centered at the zone corner. With enhancement, this peak is high enough to lead to magnetic ordering, which would then be of spin-density-wave (SDW) type.

As mentioned, magnetic order is observed experimentally for undoped $\mathrm{LaFeAsO}$ [4], and also for many but not all of the other compounds when undoped, one exception being FeSe [27]. This is already different from the cuprates. The cuprates show antiferromagnetism and insulating behavior in all the undoped compounds, and superconductivity does not exist without doping. In contrast, the Fe-based superconductors show a competition between antiferromagnetic ordering and superconductivity, but there are a variety of ways of suppressing the magnetic order - doping as in the cuprates, but also isovalent alloying on the Fe or ligand sites, pressure etc. - and all of these lead to superconductivity. The particular magnetic order that is observed in proximity to the superconducting phases is that shown in Fig. 1, i.e. that corresponding to a zone corner instability, with a doubling of the unit cell as shown.

Another related difference from the cuprates is the intimate connection between the antiferromagnetic and superconducting phases. Ning and co-workers [28] measured the NMR relaxation rate as a function of temperature for a series of samples with different doping levels. They see excess relaxation due to spin fluctuations that grows as $T$ is reduced, and which evolves continuously going from undoped, antiferromagnetic samples, to fully doped superconducting samples. Finally, the nature of the antiferromagnetic phases of the cuprates and Fe-based superconductors is very different. As mentioned, the antiferromagnetic phases of the cuprates are the Mott insulators. In contrast, the antiferromagnetic phases of the Fe-based superconductors are unambiguously metals, as seen both by bands dispersing through the Fermi energy in ARPES [29], and the observation of quantum oscillations in the magnetic phase [30].

We note that there has been some debate about the origin of the magnetic phase in the Fe-based superconductors, specifically whether the instability is driven by the Fermi surface nesting or by short range superexchange interactions. This point has been discussed in detail by Johannes and Mazin [31], who argued that the moment formation in the Fe-based superconductors is largely driven by on-site Hund's coupling and that the inter-site interactions are mediated both by electronic states close to $E_{\mathrm{F}}$, as in an SDW, and by deeper states. This was based on density functional calculations for various materials using the experimental crystal structures. One problem with this is that such calculations strongly overestimate the magnetic moments of these materials, probably because of beyond mean field renormalizations by spin fluctuations [32]. In any case, this characterization does capture the main features, specifically (1) the SDW type order strongly reconstructs the Fermi surface [29, 30, 33], (2) there are also rearrangements of bands away from $E_{\mathrm{F}}$, and (3) other orders are seen in this family of materials such as in $\mathrm{TlFe}_{2} \mathrm{Se}_{2}$ and FeTe $[34,35]$.

\section{Spin fluctuations and superconductivity}

As mentioned, Fe-based superconductivity cannot be explained by a standard electron-phonon mechanism. Therefore, other mechanisms need to be considered. Since the materials are in proximity to magnetism, it is natural to ask whether spin-fluctuation induced pairing is responsible. As shown by Berk and Schrieffer in the context of $\mathrm{Pd}$, spin fluctuations are a repulsive interaction in a singlet channel. This means that they can only stabilize a superconducting state that has sign changes in the order parameter over the Fermi surface.

The pairing interaction due to spin fluctuations is closely related to the real part of the susceptibility $\chi$ and is negative $[3,36]$. As seen in Fig. 3, this pairing interaction will be strong and negative at the antiferromagnetic 
wave vector, which is the vector connecting the electron and hole Fermi surfaces. This then favors an order parameter that changes sign between these sheets. This interaction will be highly unfavorable for a pairing channel where the order parameter on the electron and hole sheets has the same sign, much in the same way that nearness to ferromagnetism is unfavorable for standard $s$-wave superconductivity in $\mathrm{Pd}$. This observation led to the prediction of a sign changing $s$-wave order parameter in the Fe-based superconductors by Mazin and co-workers [26], and subsequently by Kuroki and co-workers [37]. This sign changing $s$-wave state, denoted $s_{ \pm}$, has average order parameters of opposite sign on the electron and hole sheets of the Fermi surface but has the same symmetry as a standard $s$-wave state.

It should be noted that while this state has on average opposite order parameters on the two sheets and could be fully gapped, it is not necessarily nodeless [38, 39]. In fact, even simple Coulomb repulsion can favor a state where there are accidental nodes on the Fermi surface. From an experimental perspective the simplest way to distinguish the $s_{ \pm}$from a standard $s$-wave state or from other states such as $d$-wave is through coherence factors. One is the NMR Hebel-Slichter peak which is suppressed with an $s_{ \pm}$order parameter but not with a standard $s$-wave, and another is a neutron spin resonance, which should occur for this state at the $2 \mathrm{D}$ nesting vector, $(\pi, \pi)$ $[40,41]$.

This resonance has been observed at the nesting wave vector showing that there is a sign change between the Fermi surface sections separated by it, i.e. as expected in the $s_{ \pm}$case [42-45]. Interestingly, in doped $\mathrm{BaFe}_{2} \mathrm{As}_{2}$, which has a noticeable corrugation of the Fermi surface along the $k_{z}$ direction [46], the resonance also shows $k_{z}$ dependence depending on doping and presumably reflecting $k_{z}$ dispersion of the spin-fluctuations [43, 44].

Within a mean field picture, SDW magnetic ordering will occur when the bare (non-enhanced) susceptibility, $\operatorname{Re}\left(\chi_{0}\right)$ exceeds a threshold value at the ordering wave vector, $\boldsymbol{q}$, so that the RPA enhanced susceptibility, $\chi(\boldsymbol{q})=\chi_{0}(\boldsymbol{q}) /\left[1-I(\boldsymbol{q}) \chi_{0}(\boldsymbol{q})\right]$, diverges. What matters therefore is the magnitude at a specific $\boldsymbol{q}$. Beyond the RPA level, spin fluctuations work against ordering. The extent of this suppression is related to an integral of the imaginary part of the susceptibility over wave vector and energy by the fluctuation dissipation theorem [47, 48]. Qualitatively, this reflects the intuitive result that competition between different magnetic states works against ordering. As such, for a given peak value of $\chi_{0}$, a sharp peak is more favorable for magnetic ordering than a broad peak such as that shown in Fig. 2.

In contrast, the BCS gap equation involves an integral of the order parameter with the pairing interaction (related to $\operatorname{Re}(\chi))$, i.e. for the $s_{ \pm}$state, the integral of over the region of $\boldsymbol{q}$ that can connect the electron and hole Fermi surfaces (a region set by the Fermi surface size). Thus, for superconductivity a broad peak with a large weight is much better than a narrow peak with a smaller weight. In other words, competition between different related magnetic states is favorable for superconductivity. In fact, it is doubly favorable, because it also suppresses the competing phase, i.e. SDW order.

\section{Magnetism}

The occurrence of the zone corner magnetic instability (Fig. 1) in iron-based superconductors is associated with the nested Fermi surface of these materials. When the nesting is removed, as it is by overdoping in $\mathrm{TlFe}_{2} \mathrm{Se}_{2}$, other magnetic orders appear [34]. Significantly, these are quite stable. In $\mathrm{TlFe}_{2} \mathrm{Se}_{2}$ the reported Néel temperature is $\approx 450 \mathrm{~K}$ [49], i.e. higher than the SDW type ordering in the undoped compounds. When the nesting is present, as it is over most of the chemically stable range of these compounds, one may have SDW ordering, superconductivity, a co-existence of SDW ordering and superconductivity or a different magnetic order as in FeTe. This underscores the competition between different states.

The SDW order occurs with various magnetic moments ranging from zero up to $\approx 1 \mu_{\mathrm{B}} / \mathrm{Fe}$ depending on the compound and doping level. This is in contrast to the expected behavior for a local moment system. Furthermore, these materials do not show Curie-Weiss behavior above Néel temperature. In contrast, measurements show $\chi(T)$ that is an increasing function of temperature up to high temperature for both undoped, antiferromagnetic material and doped superconducting samples [50]. This can be interpreted as a temperature dependent break up of antiferromagnetic correlations, indicative of a magnetic energy scale that is much higher than the actual ordering temperature.

Standard density functional theory (DFT) calculations show large errors in the description of the magnetic properties of these compounds. In particular, these calculations, which effectively do not include renormalization due to spin fluctuations [51], yield much larger moments than experimentally observed, including prediction of magnetism for materials that are superconducting and not magnetically ordered [32].

The comparison of DFT results and experiment also reveals in interesting interplay between bonding and moment formation: calculations with magnetism reproduce the experimental $\mathrm{Fe}-\mathrm{As}$ bond lengths at the expense of very overestimated moments, while calculations without moments (as is the case for the doped superconducting phases) yield bond lengths that are $\approx 0.1 \AA$ too short a large error for modern DFT calculations. The inference that has been drawn from this $[10,32,34]$ is that from the point of view of bonding these materials have moments (bonding is at the $\mathrm{eV}, 10^{14} \mathrm{~s}^{-1}$ scale), while on longer time scales the moments are strongly renormalized by spin-fluctuations.

Other evidence for strong spin fluctuations in these materials comes from transport measurements that show a strong reduction in the resistivity as $T$ is lowered through 
the ordering transition [52], even though much of the Fermi surface is gapped in the SDW state. This indicates a very strong reduction in the scattering rate in the ordered phase, and therefore strong spin fluctuation scattering at high $T$. Also, core level spectroscopy has shown substantial exchange splitting of the Fe $3 s$ core level, in doped superconducting $\operatorname{CeFeAs}(\mathrm{O}, \mathrm{F})$, even though this material has no magnetic ordering [53].

All of this suggests a rather fascinating interplay between magnetism that is suppressed by spin fluctuations and spin fluctuation induced superconductivity. We speculate that the role of these renormalizations in the Fe-based superconductors is to provide a way of having metal without magnetic order and with a high magnetic energy scale and resulting high $T_{\mathrm{c}}$ superconductivity, when such a scale would normally lead to ordered magnetism instead, as in the $450 \mathrm{~K}$ magnetism of $\mathrm{TlFe}_{2} \mathrm{Se}_{2}$.

\section{Summary}

The iron-based superconductors are a new family of high $T_{\mathrm{c}}$ materials that seem in many respects to be different from the cuprates. The most striking difference is the apparently moderately correlated nature of the Fe-based compounds in contrast to the strong correlated behavior of the cuprates, which are the only other class of materials with $T_{\mathrm{c}}$ above $50 \mathrm{~K}$. At this point it is not clear whether Fe-based superconductivity shares the same origin as the high $T_{\mathrm{c}}$ superconductivity of the cuprates. It may be that future work on these materials will reveal the essential similarity of them and the mechanism of superconductivity in both or it may be that they are essentially different and nature has provided multiple routes to high $T_{\mathrm{c}}$, which are embodied in different classes of materials. In any case, experimental data and comparison of experiment and DFT calculations show evidence for exceptionally strong renormalization of the magnetism of these compounds by spin fluctuations. However, at this time there is not a quantitative first principles theory for calculating such renormalizations. Developing such a theory is an important challenge.

\section{Acknowledgments}

This work was supported by the U.S. Department of Energy, Basic Energy Sciences, Materials Sciences and Engineering Division.

\section{References}

[1] Y. Kamihara, H. Hiramatsu, M. Hirano, R. Kawamura, H. Yanagi, T. Kamiya, H. Hosono, J. Am. Chem. Soc. 128, 10012 (2006).

[2] Y. Kamihara, T. Watanabe, M. Hirano, H. Hosono, J. Am. Chem. Soc. 130, 3296 (2008).

[3] N.F. Berk, J.R. Schrieffer, Phys. Rev. Lett. 17, 433 (1966).
[4] C. de la Cruz, Q. Huang, J.W. Lynn, J.Y. Li, W. Ratcliff II, J.L. Zaretsky, H.A. Mook, G.F. Chen, J.L. Luo, N.L. Wang, P.C. Dai, Nature (London) 453, 899 (2008).

[5] M. Rotter, M. Tegel, D. Johrendt, I. Schellenberg, W. Hermes, R. Pottgen, Phys. Rev. B 78, 020503 (2008).

[6] D.J. Singh, M.H. Du, Phys. Rev. Lett. 100, 237003 (2008).

[7] L. Boeri, O.V. Dolgov, A.A. Golubov, Phys. Rev. Lett. 101, 026403 (2008).

[8] A.S. Sefat, D.J. Singh, MRS Bull. 36, 614 (2011).

[9] S. Lebegue, Phys. Rev. B 75, 035110 (2007).

[10] D.J. Singh, Physica C 469, 418 (2009).

[11] A. Koitzsch, D. Inosov, J. Fink, M. Knupfer, H. Eschrig, S.V. Borisenko, G. Behr, A. Kohler, J. Werner, B. Buchner, R. Follath, H.A. Durr, Phys. Rev. B 78, 180506 (2008).

[12] O. Gunnarsson, E. Koch, R.M. Martin, Phys. Rev. B 56, 1146 (1997).

[13] E.Z. Kurmaev, R.G. Wilks, A. Moewes, N.A. Skorikov, Y.A. Izyumov, L.D. Finkelstein, R.H. Li, X.H. Chen, Phys. Rev. B 78, 220503 (2008).

[14] D.H. Lu, M. Yi, S.K. Mo, A.S. Erickson, J. Analytis, J.C. Chu, D.J. Singh, Z. Hussain, T.H. Geballe, I.R. Fisher, Z.X. Shen, Nature (London) 455, 81 (2008).

[15] M.M. Qazilbash, J.J. Hamlin, R.E. Baumbach, L. Zhang, D.J. Singh, M.B. Maple, D.N. Basov, $\mathrm{Na}_{-}$ ture Phys. 5, 647 (2009).

[16] A. Liebsch, Phys. Rev. B 84, 180505(R) (2011).

[17] A.S. Sefat, R. Jin, M.A. McGuire, B.C. Sales, D.J. Singh, D. Mandrus, Phys. Rev. Lett. 101, 117004 (2008).

[18] A. Leithe-Jasper, W. Schnelle, C. Geibel, H. Rosner, Phys. Rev. Lett. 101, 207004 (2008).

[19] W. Schnelle, A. Leithe-Jasper, R. Gemeniuk, U. Burkhardt, D. Kasinathan, H. Rosner, Phys. Rev. B 79, 214516 (2009).

[20] L. Zhao, H. Liu, W. Zhang, J. Meng, X. Jia, G. Liu, X. Dong, G.F. Chen, J.L. Luo, G. Wang, Y. Zhou, Y. Zhu, X. Wang, Z. Zhao, Z. Xu, C. Chen, X.J. Zhou, Chin. Phys. Lett. 25, 4402 (2008).

[21] T. Kondo, A.F. Santander-Syro, O. Copie, C. Liu, M.F. Tilman, E.D. Mun, J. Schmalian, S.L. Budko, M.A. Tanatar, P.C. Canfield, A. Kaminski, Phys. Rev. Lett. 101, 147003 (2008).

[22] C. Liu, G.D. Samolyuk, Y. Lee, N. Ni, T. Kondo, A.F. Santander-Syro, S.L. Budko, J.L. McChesney, E. Rotenberg, T. Valla, A.V. Fedorov, P.C. Canfield, B.N. Harmon, A. Kaminski, Phys. Rev. Lett. 101, 177005 (2008).

[23] M. Yi, D.H. Lu, J.G. Analytis, J.H. Chu, S.K. Mo, R.H. He, R.G. Moore, X.J. Zhou, G.F. Chen, J.L. Luo, N.L. Wang, Z. Hussain, D.J. Singh, I.R. Fisher, Z.X. Shen, Phys. Rev. B 80, 024515 (2009). 
[24] H. Ding, P. Richard, K. Nakayama, K. Sugawara, T. Arakane, Y. Sekiba, A. Takayama, S. Souma, T. Sato, T. Takahashi, Z. Wang, X. Dai, Z. Fang, G.F. Chen, J.L. Luo, N.L. Wang, Europhys. Lett. 83, 47001 (2008).

[25] L. Wray, D. Qian, D. Hsieh, Y. Xia, L. Li, J.G. Checkelsky, A. Pasupathy, K.K. Gomes, C.V. Parker, A.V. Fedorov, G.F. Chen, J.L. Luo, A. Yazdani, N.P. Ong, N.L. Wang, M.Z. Hasan, Phys. Rev. B 78, 184508 (2008).

[26] I.I. Mazin, D.J. Singh, M.D. Johannes, M.H. Du, Phys. Rev. Lett. 101, 057003 (2008).

[27] S. Medvedev, T.M. McQueen, I.A. Toryan, T. Palasyuk, M.I. Eremets, R.J. Cava, S. Naghavi, F. Casper, V. Ksenofontov, G. Wortmann, C. Felser, Nature Mater. 8, 630 (2009).

[28] F. Ning, K. Ahlian, T. Imai, A.S. Sefat, R. Jin, M.A. McGuire, B.C. Sales, D. Mandrus, J. Phys. Soc. Jpn. 78, 013711 (2009).

[29] M. Yi, D.H. Lu, J.G. Analytis, J.H. Chu, S.K. Mo, R.H. He, M. Hashimoto, R.G. Moore, I.I. Mazin, D.J. Singh, Z. Hussain, I.R. Fisher, Z.X. Shen, Phys. Rev. B 80, 174510 (2009).

[30] S.E. Sebastian, J. Gillett, N. Harrison, P.H.C. Lau, D.J. Singh, C.H. Mielke, G.G. Lonzarich, J. Phys. Condens. Matter 20, 422203 (2008).

[31] M.D. Johannes, I.I. Mazin, Phys. Rev. B $\mathbf{7 9 ,}$ 22051(R) (2009).

[32] I.I. Mazin, M.D. Johannes, L. Boeri, K. Koepernik, D.J. Singh, Phys. Rev. B 78, 085104 (2008).

[33] W.Z. Hu, J. Dong, G. Li, Z. Li, P. Zheng, G.F. Chen, J.L. Luo, N.L. Wang, Phys. Rev. Lett. 101, 257005 (2008).

[34] L. Zhang, D.J. Singh, Phys. Rev. B 79, 094528 (2009).

[35] S. Li, C. de la Cruz, Q. Huang, Y. Chen, J.W. Lynn, J. Hu, Y.L. Huang, F.C. Hsu, K.W. Yeh, M.K. Wu, P. Dai, Phys. Rev. B 79, 054503 (2009).

[36] T. Moriya, K. Ueda, Rep. Prog. Phys. 66, 1299 (2003).

[37] K. Kuroki, S. Onari, R. Arita, H. Usui, Y. Tanaka, H. Kotani, H. Aoki, Phys. Rev. Lett. 101, 087004 (2008).

[38] A.V. Chubukov, M.G. Vavilov, A.B. Vorontsov, Phys. Rev. B 80, 140515 (2009).

[39] S. Maiti, A.V. Chubukov, Phys. Rev. B 82, 214515 (2010).
[40] T. Maier, D.J. Scalapino, Phys. Rev. B 78, 020514(R) (2008).

[41] M. Korshunov, I. Eremin, Phys. Rev. B $\mathbf{7 8 ,}$ 140509(R) (2008).

[42] A.D. Christianson, E.A. Goremychkin, R. Osborn, S. Rosenkranz, M.D. Lumsden, C.D. Malliakas, I.S. Todorov, H. Claus, D.Y. Chung, M.G. Kanatzidis, R.I. Bewley, T. Guidi, Nature (London) 456, 930 (2008).

[43] S. Chi, A. Schneidewind, J. Zhao, L.W. Harriger, L. Li, Y. Luo, G. Cao, Z. Xu, M. Loewenhaupt, J. Hu, P. Dai, Phys. Rev. Lett. 102, 107006 (2009).

[44] D.K. Pratt, A. Kreyssig, S. Nandi, N. Ni, A. Thaler, M.D. Lumsden, W. Tian, J.L. Zarestky, S.L. Budko, P.C. Canfield, A.I. Goldman, R.J. McQueeney, Phys. Rev. B 81, 140510(R) (2010).

[45] D.S. Inosov, J.T. Park, P. Bourges, D.L. Sun, Y. Sidis A. Schneidewind, K. Hradil, D. Haug, C.T. Lin, B. Keimer, V. Hinkov, Nature Phys. 6, 178 (2010).

[46] P. Vilmercati, A. Fedorov, I. Vobornik, U. Manju, G. Panaccione, A. Goldini, A.S. Sefat, M.A. McGuire, B.C. Sales, R. Jin, D. Mandrus, D.J. Singh, N. Mannella, Phys. Rev. B 79, 220503(R) (2009).

[47] M. Shimizu, Rep. Prog. Phys. 44, 329 (1981).

[48] T. Moriya, Spin Fluctuations in Itinerant Electron Magnetism, Springer, Berlin 1985.

[49] L. Haggstrom, H.R. Verma, S. Bjarman, R. Wappling, R. Berger, J. Solid State Chem. 63, 401 (1986).

[50] R. Klingeler, N. Leps, I. Hellmann, A. Popa, U. Stockert, C. Hess, V. Kataev, H.J. Grafe, F. Hammerath, G. Lang, S. Wormehl, G. Behr, L. Harnagea, S. Singh, B. Buchner, Phys. Rev. B 81, 024506 (2010).

[51] A. Aguayo, I.I. Mazin, D.J. Singh, Phys. Rev. Lett. 92, 147201 (2004).

[52] M.A. McGuire, A.D. Christianson, A.S. Sefat, B.C. Sales, M.D. Lumsden, R. Jin, E.A. Payzant, D. Mandrus, Y. Luan, V. Keppens, V. Varadarajan, J.W. Brill, R.P. Hermann, M.T. Sougrati, F. Grandjean, G.J. Long, Phys. Rev. B 78, 094517 (2008).

[53] F. Bondino, E. Magnano, M. Malvestuto, F. Parmigiani, M.A. McGuire, A.S. Sefat, B.C. Sales, R. Jin, D. Mandrus, E.W. Plummer, D.J. Singh, N. Mannella, Phys. Rev. Lett. 101, 267001 (2008). 